\title{
Management of psoriasis in children (Review)
}

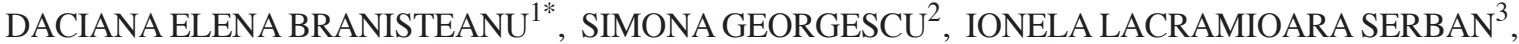 \\ ALIN CONSTANTIN PINZARIU ${ }^{3}$, DANIEL BODA ${ }^{2}$, MINELA AIDA MARANDUCA ${ }^{3}$, \\ MIHAI GLOD ${ }^{4 *}$, CATALINA IOANA BRANISTEANU $^{5 *}$, ROXANA BILIBAU $^{6}$, ANDREEA DIMITRIU $^{7}$, \\ ALIN CODRUT NICOLESCU ${ }^{8}$ and DANIEL CONSTANTIN BRANISTEANU ${ }^{9}$
}

\author{
${ }^{1}$ Department of Dermatology, ‘Grigore T. Popa’ University of Medicine and Pharmacy, 700115 Iasi; \\ ${ }^{2}$ Department of Dermatology, 'Carol Davila' University of Medicine and Pharmacy, 050474 Bucharest; \\ Departments of ${ }^{3}$ Physiology and ${ }^{4}$ Surgery, 'Grigore T. Popa' University of Medicine and Pharmacy; \\ ${ }^{5}$ 'Grigore T. Popa' University of Medicine and Pharmacy, 700115 Iasi; ${ }^{6}$ Department of Dermatology, \\ 'Railway Clinical Hospital', 700506 Iasi; ${ }^{7}$ Department of Dermatology, 'Arcadia Hospitals and Medical Centers', \\ 700620 Iasi; ${ }^{8}$ Department of Dermatology, Roma Medical Center for Diagnosis and Treatment, 011773 Bucharest; \\ ${ }^{9}$ Department of Ophthalmology, 'Grigore T. Popa' University of Medicine and Pharmacy, 700115 Iasi, Romania
}

Received July 27, 2021; Accepted August 26, 2021

DOI: $10.3892 / \mathrm{etm} .2021 .10864$

\begin{abstract}
Psoriasis is a common long-lasting, inflammatory disease that mainly affects the skin. The incidence of this condition has increased significantly over time and at this point, it affects approximately $1 \%$ of children. Psoriasis can appear at any age, including childhood and adolescence, with a higher frequency in girls, an earlier onset being associated with severe psoriasis. The pathology is the result of the interaction between genetics and trigger factors such as infections, stress, diet, obesity, and chemical irritants. Paradoxically, tumor necrosis factor (TNF)- $\alpha$ inhibitors (infliximab, adalimumab) may induce psoriasis in children. Psoriasis is a long-term condition with periods of exacerbation; thus, the quality of life can be affected and patients should receive psychosocial support. Although most children have mild disease and topical treatment is efficient, some cases are challenging to treat. The aim of this review was to provide an overview of the current knowledge concerning the epidemiology, etiology, pathogenesis, clinical features, comorbidities, and treatment of psoriasis in children and also to emphasize the need for a multidisciplinary approach to this complex pathology.
\end{abstract}

Correspondence to: Professor Ionela Lacramioara Serban, Department of Physiology, 'Grigore T. Popa' University of Medicine and Pharmacy, 16 Universitatii Street, 700115 Iasi, Romania

E-mail: ionela.serban@umfiasi.ro

Dr Roxana Bilibau, Department of Dermatology, 'Railway Clinical Hospital', 1 Garabet Ibraileanu Street, 700506 Iasi, Romania

E-mail: bilibau.roxana@gmail.com

${ }^{*}$ Contributed equally

Key words: psoriasis in children, management, napkin psoriasis, biologic therapies, pathogenesis, treatment

\section{Contents}

1. Introduction

2. Epidemiology

3. Pathogenesis

4. Clinical features

5. Comorbidities

6. Treatment

7. Conclusions

\section{Introduction}

Psoriasis is a chronic, systemic, inflammatory, immune-mediated disease that primarily affects the skin, characterized by abnormal hyperproliferation of the epidermis (1-3). There are many trigger factors involved (stress, infections, medications, trauma) that can induce the disease in a predisposed population (3).

To collect reports of psoriasis etiopathogeny, a literature search was conducted using electronic databases Google Scholar, PubMed, Key Elsevier, UpToDate and Medscape for the terms 'psoriasis' in combination with 'psoriasis in children', 'management of psoriasis', 'napkin psoriasis', 'biologic therapies', 'pathogenesis', 'treatment'. Our study includes case reports, case series, and literature review-type papers. Based on 63 publications found in the literature, we compiled a concised report, illustrated with images from individual patients. A parental written informed consent before the publication of de-identified patient photographs was obtained.

\section{Epidemiology}

Psoriasis is the second most frequent disorder after atopic dermatitis in childhood and affects approximately $1 \%$ of children $(4,5)$. The incidence of childhood psoriasis has increased dramatically over time from 29.6 per 100,000 individuals in 1970-1974 to 62.7 per 100,000 in 1995-1999 (6). The disease can appear at 
any age, more commonly at 9-10 years, and has a durable course with exacerbation periods that can last between a few weeks and 1.5 years (7). The incidence of pediatric psoriasis increases with age. In the UK, the prevalence was found to increase from $0.55 \%$ in children between $0-9$ years of age to $1.37 \%$ in children between 10-19 years $(8,9)$. In Italy, the prevalence of psoriasis in children is estimated at $2.1 \%$ (10). The exacerbations are more likely to appear in the autumn and winter seasons (7). Psoriasis appears earlier and more frequently in girls. Earlier onset is also associated with severe forms of psoriasis $(11,12)$.

\section{Pathogenesis}

Psoriasis is the result of polygenic predisposition and environmental triggers. Genetic predisposition is easier to detect in children than in adults. Family history is present in 35 to $90 \%$ of patients with psoriasis, of which $30 \%$ are first-degree relatives and the pathology is more common in monozygotic twins compared to dizygotic twins $(3,13)$. Family history has been associated with earlier onset of psoriasis and the presence of enthesitis (14). There are over 1,300 susceptibility genes identified in psoriatic skin which are differently expressed compared to normal skin (15). The most important psoriasis-susceptibility gene (PSORS1) is human leukocyte antigen HLA Cw6 (16). The presence of this gene is associated with a relative risk of developing psoriasis of $13 \%$ for Caucasians and $25 \%$ for the Japanese population. Based on this, some clinicians consider that there are two types of psoriasis, type I with early-onset, positive family history, and the presence of HLA Cw6 and type II with late-onset, without family history and no expression of HLA Cw6 (3). The corneodesmosin $(C D S N)$ gene has been associated with psoriasis in the Caucasian population. Other genes involved are the MHC class I polypeptide-related sequence A (MICA) gene, according to a study of psoriasis in the Chinese population, and the angiotensin I converting enzyme (ACE) gene in some patients (1). Promoter region polymorphisms in the tumor necrosis factor $(T N F) \alpha$ gene are associated with psoriasis in the Polish population and interleukin 12B (IL12B) gene polymorphisms are found in different patients (1). In addition, protein tyrosine phosphatase, non-receptor type 22 (PTPN22) region polymorphisms are related to early-onset psoriasis (1).

The pathogenesis of psoriasis is based on chronic inflammation, increased keratinocyte proliferation, and dysfunctional differentiation. Although the main changes in psoriasis are found in the most superficial layer of the skin, formed by keratinocytes, the psoriatic plaque is not limited to inflammation of the epidermis. It is characterized by the interaction between keratinocytes and many different cells, extending to the dermal layer of the skin (17-19). The factors that have a key role in the pathogenesis of psoriasis are T cells, keratinocytes, Langerhans' cells, macrophages, and some types of cytokines. Self-antigens activate dendritic cells and release cytokines including TNF, $\gamma$-interferon, interleukin(IL)-12, and IL-23 that recruit T cells, Th-1, Th-17 and Th-22 (2). T cells release cytokines and maintain the abnormal differentiation of keratinocytes and cell cycle turnover $(16,20)$. Keratinocytes from psoriatic plaque express signal transducer and activator of transcription 3 (STAT3), a transcription factor that was found to produce psoriasis-like injuries in mice. The fact that STAT3 is activated by cytokines (IL-6, IL-20, IL-22) could explain the connection between keratinocyte proliferation and the immune system in the pathogenesis of psoriasis (3). There are several vascular changes such as angiogenesis [stimulated by IL- 8 and transforming growth factor (TGF)- $\alpha$ ], dilated sinuous vessels in the papillary dermis, and the formation of high endothelial venules. Therefore, the size of the microcirculation expands and facilitates the passage of $\mathrm{T}$ lymphocytes ( $\mathrm{T}$ helper 1 subclass), maintaining psoriatic plaque. Severe psoriasis is accompanied by microvascular hyperpermeability mediated by vascular endothelial growth factor (VEGF) (1).

The pathogenesis of psoriasis can be structured in an initiation phase possibly caused by trauma, infections, or drugs, and a chronic phase with long-term clinical progression. The main trigger factors in children include stress and infections, especially streptococcal infections $(3,5,21)$. Some possible causes of psoriasis morbidity in children are social and home problems, emotional issues, pollution, and a decrease in immune reactivity (5). In addition, overweight and obesity have been recently discovered as risk factors for psoriasis in children (2). Paradoxically, TNF inhibitors such as infliximab and adalimumab may induce psoriasis (4).

\section{Clinical features}

The history and symptoms of psoriasis can be different according to the age of children and the type of psoriasis. Most common psoriasis lesions are found in the extremities $(60 \%)$ and the scalp (47\%) (6). The most common clinical manifestation is pruritus (22). In many cases, infants have persistent diaper rash that remains despite many treatments. Older children may have an asymptomatic scaly rash or severe dandruff (2). Adolescents have the same clinical features as adults. Some children may present the Koebner phenomenon that consists in the appearance of new skin lesions on previously unaffected skin secondary to trauma, lesions that are pronounced and long-lasting $(2,5)$.

Psoriasis is characterized by erythematous papules that merge to create well-demarcated plaques with irregular borders, covered by a silvery-white scale. If the scale is removed, pinpoint bleeding will result, known as the Auspitz sign. Children with psoriasis have more facial and flexural lesions and the plaques are smaller and thinner compared to adults $(2,23)$ (Figs. 1 and 2). The differential diagnosis is often difficult to make with seborrheic dermatitis $(2,16)$.

There are several types of psoriasis: vulgaris: chronic plaque (Fig. 3), guttate (Fig. 4), and inverse psoriasis (Fig. 5), pustular and erythrodermic.

The most common type of psoriasis in children is chronic plaque psoriasis (75\% of children with psoriasis) followed by guttate psoriasis $(15-30 \%$ of children with psoriasis) $(2,24)$. In addition, diaper psoriasis is a common variant exclusively seen in infants (Figs. 6-8). Chronic plaque psoriasis is most common on the extensor extremities, trunk, flexures, and face. Guttate psoriasis is characterized by the sudden onset of droplet papules with symmetrical disposal over the trunk, limbs, and face (Fig. 9). These lesions may be preceded by streptococcal infection and resorb within 3 to 4 weeks or they can persist and transform into severe psoriasis (2). The development of lesions in flexural areas and the face is more common in children and is known as inverse psoriasis. The psoriasis of the scalp 


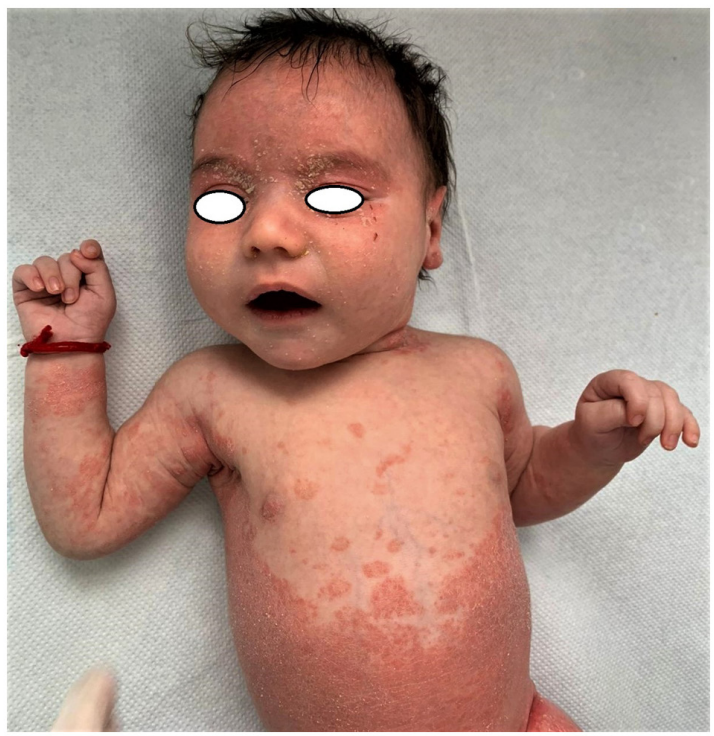

Figure 1. Facial lesions in napkin psoriasis.

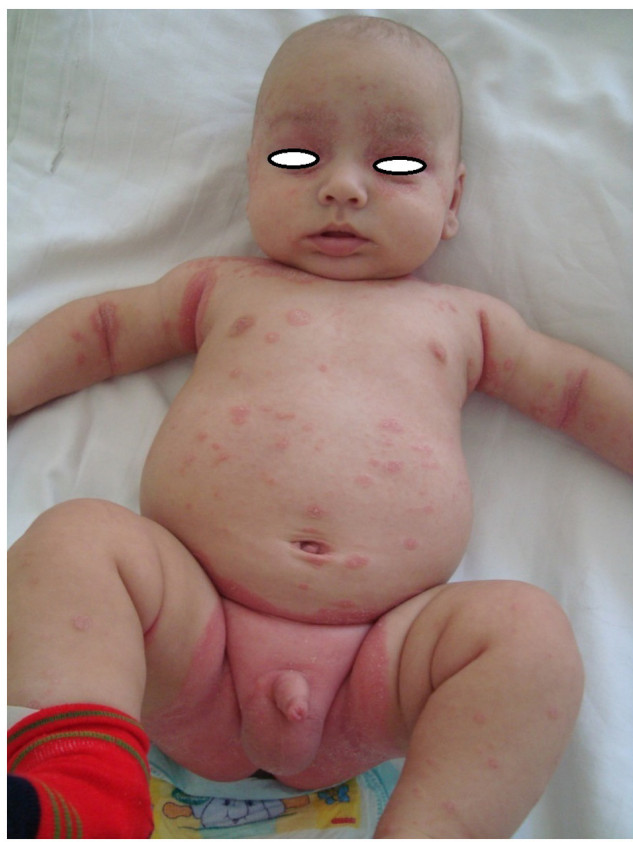

Figure 2. Facial and flexular lesions in napkin psoriasis

has well-defined, scaly, erythematous, and pruritic lesions. In children with alopecia, the hair grows back when the disease is under control (2). Nail psoriasis is less frequent in children compared to adults but can be observed in approximately $40 \%$ of the children. The most common changes are pitting, leukonychia, and longitudinal ridges that are very difficult to treat $(2,25,26)$ (Fig. 10). Pustular psoriasis is rare and consists of sterile superficial pustules with annular disposition (Fig. 11). If pustules are generalized, children may develop a fever and they may be often mistaken for an infection. Another variant of this disease is erythrodermic psoriasis (Fig. 12) that affects more than $90 \%$ of the body surface and is extremely rare $(2,16)$.

The severity of skin disease is classified based on the affected body surface area (BSA) According to this, the

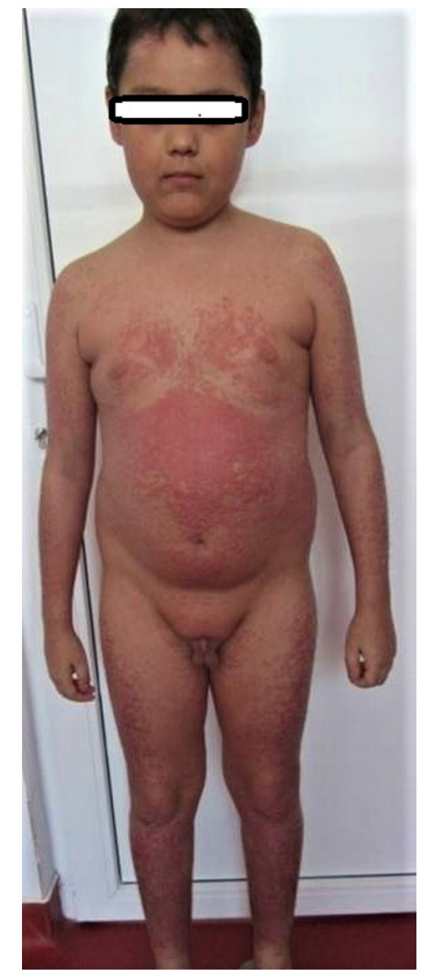

Figure 3. Chronic plaque psoriasis.

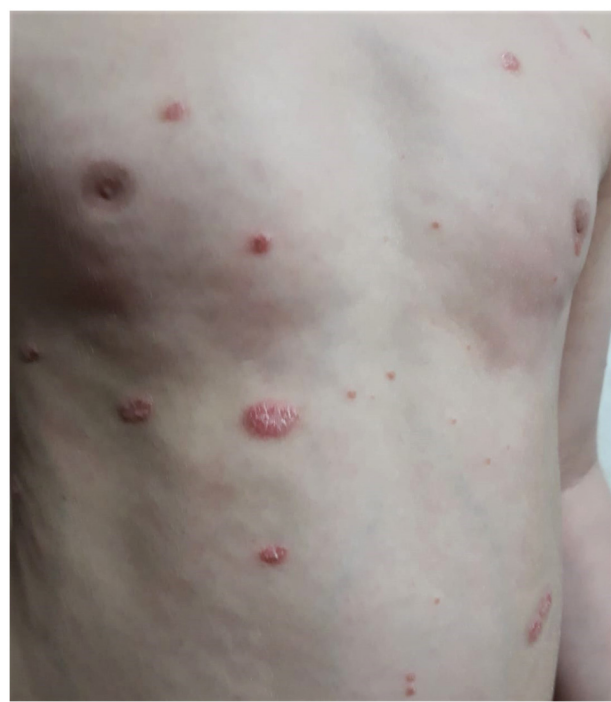

Figure 4. Guttate psoriasis.

disease is mild if lesions cover less than $5 \%$ of BSA, moderate if it affects between 5 and $10 \%$ of BSA and severe if the BSA involved is greater than $10 \%$ (16). Fortunately, most children with psoriasis have mild disease $(2,22)$.

\section{Comorbidities}

Some recent studies have discovered that children with psoriasis are twice as likely to have comorbidities as those without psoriasis (2). Psoriasis has been associated with type 1 diabetes, rheumatoid arthritis, Crohn's disease, systemic lupus erythematosus, vitiligo (Fig. 13), alopecia areata, eczema, and 


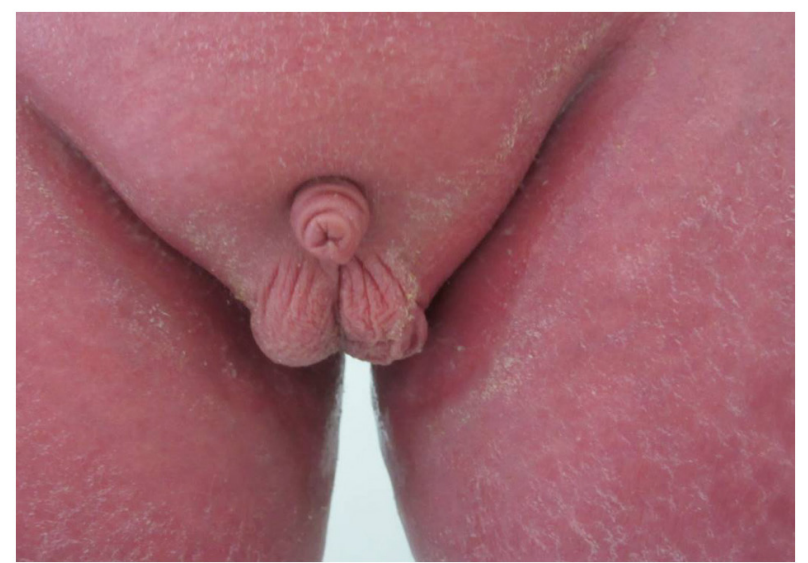

Figure 5. Inverse psoriasis.

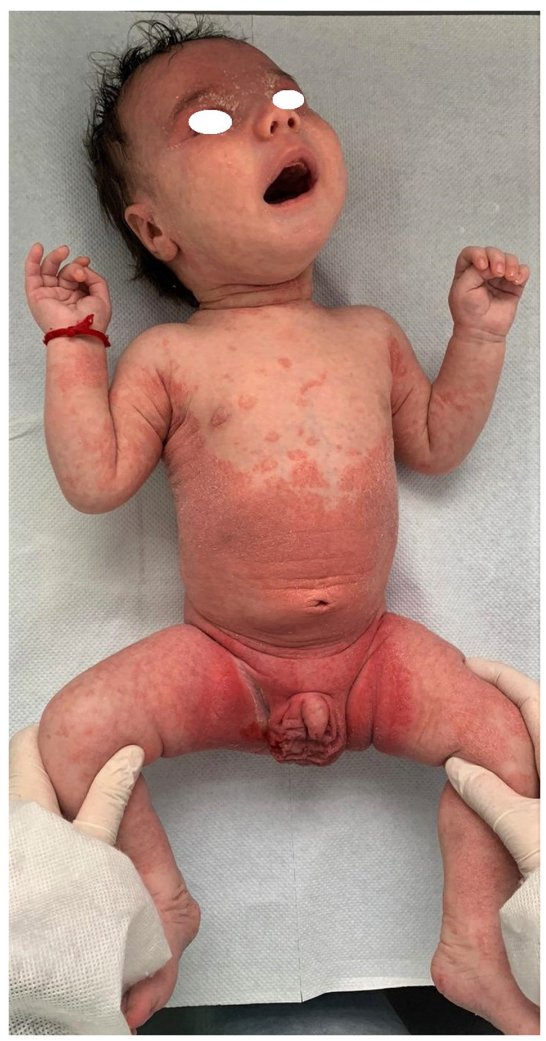

Figure 6. Napkin psoriasis.

lichen planus $(2,27,28)$. Psoriasis can be mistaken with seborrheic dermatitis and avitaminosis, but they can also coexist. Just like in adults, the association with atopic dermatitis is frequently seen in children. In terms of association with systemic diseases, children with psoriasis have a higher rate of obesity, especially boys. Patients with this comorbidity are more likely to have a severe form of psoriasis compared to normal-weight psoriatic children (29). The major comorbidity is psoriatic arthritis. Prevalence in the children population is still unknown. Psoriatic arthritis can occur at any age, but the onset is more common between 2 and 3 years and 9 to 12 years of age. This condition causes joint pain and mostly affects finger and toe joints $(30,31)$. Younger children with psoriasis, especially girls, present an onset with an oligoarticular disease

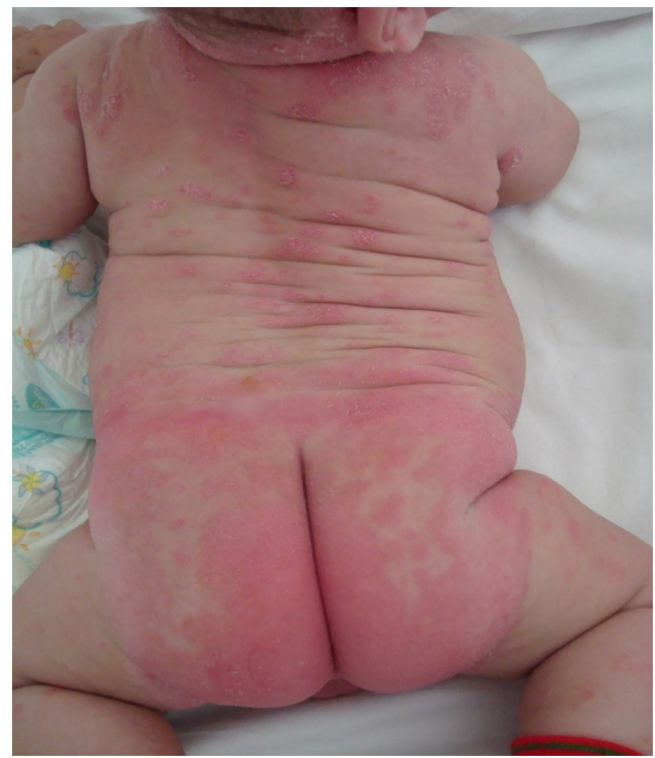

Figure 7. Napkin psoriasis.

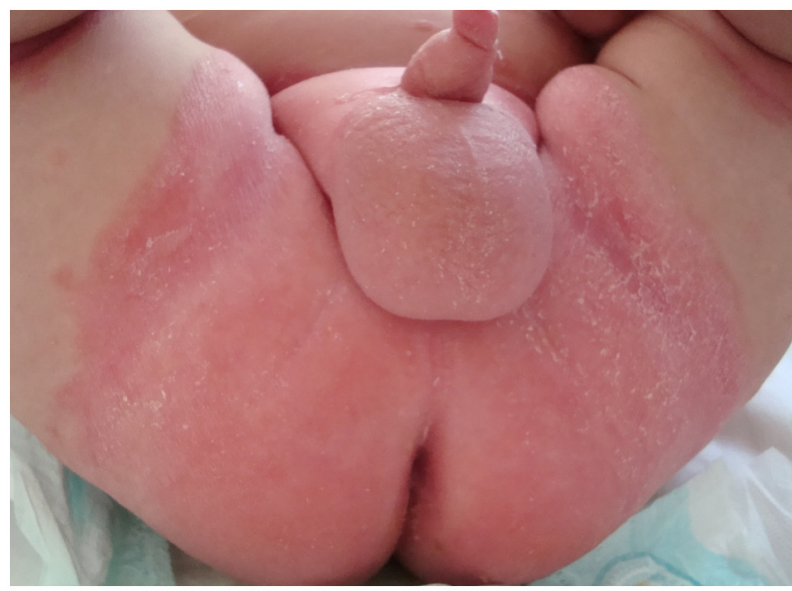

Figure 8. Napkin psoriasis.

or dactylitis while older children, especially boys, present an onset with enthesitis and axial joint involvement.

Pediatric patients with concomitant arthritis can develop uveitis (4). Children and adolescents with this condition are reported to have an increased risk of hypertension, hyperlipidemia, and diabetes, probably due to the high rate of metabolic syndrome. Low levels of HDL cholesterol and high concentrations of fasting glucose appear in the early stages of metabolic syndrome in psoriatic children (32).

The presence of skin lesions harms the children. Many patients are stigmatized by other children and recreational activity is highly affected. This stigmatization can induce changes in behavior, depression, and anxiety. More than that, the association between psoriasis and obesity increases the risk of social isolation, withdrawal, depression, and anxiety $(4,33)$. Psoriasis affects the quality of life of children and their parents and they should receive psychosocial support (34).

As in most systemic, inflammatory, chronic diseases, ocular manifestations can occur (35). The incidence of ocular involvement in psoriasis largely varies in the literature 


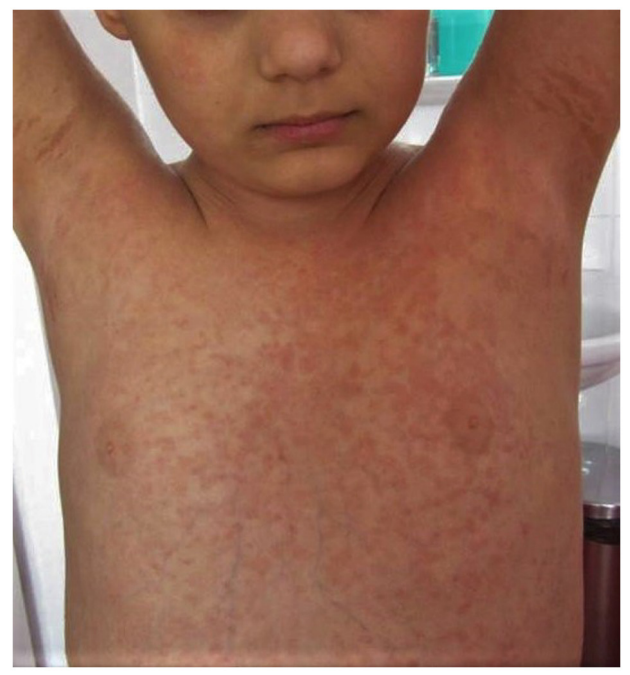

Figure 9. Guttate psoriasis.

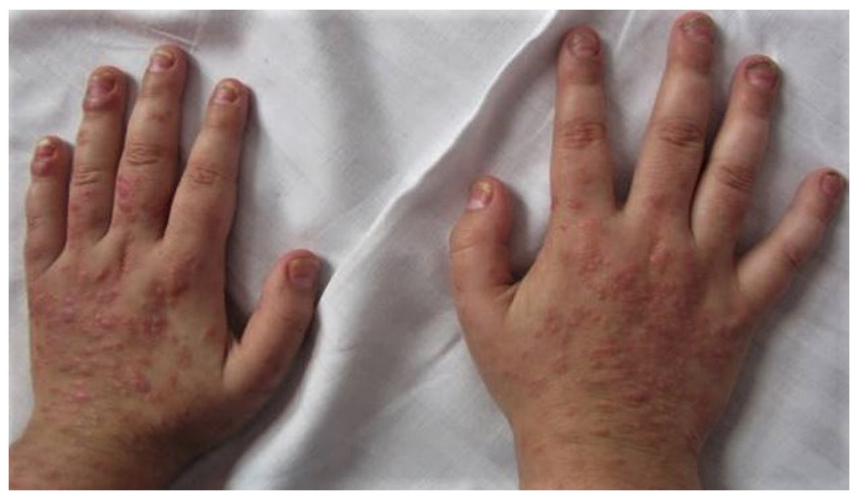

Figure 10. Nail psoriasis.

between 12 and $58 \%(36,37)$ and even up to $81.4 \%$ in more recent studies (38). The eyelid manifestations include blepharitis, which is the most frequent one, together with erythema, psoriatic plaques, and edema leading to eyelid abnormalities such as ectropion, trichiasis, and madarosis. In moderate and severe forms of the disease, chronic conjunctivitis can lead to limbal lesions and keratoconjunctivitis sicca further responsible for corneal lesions such as punctate keratitis, recurrent erosions, opacities, and vascularization (39). The anterior uveitis associated with childhood psoriatic arthritis and onset before the age of 6 years has been described as bilateral, long-lasting, and severe requiring biologic therapy (40).

\section{Treatment}

Psoriasis therapy varies according to patient age, type of psoriasis, affected sites, and extension of the disease (16). This condition requires the involvement of several specialists such as dermatologists, pediatricians, and rheumatologist. There are 4 types of treatment as described.

Topical agents. Topical agents such as emollients, vitamin D analogs, and corticosteroids should be the first choice of treatment for children with mild to moderate psoriasis (41).

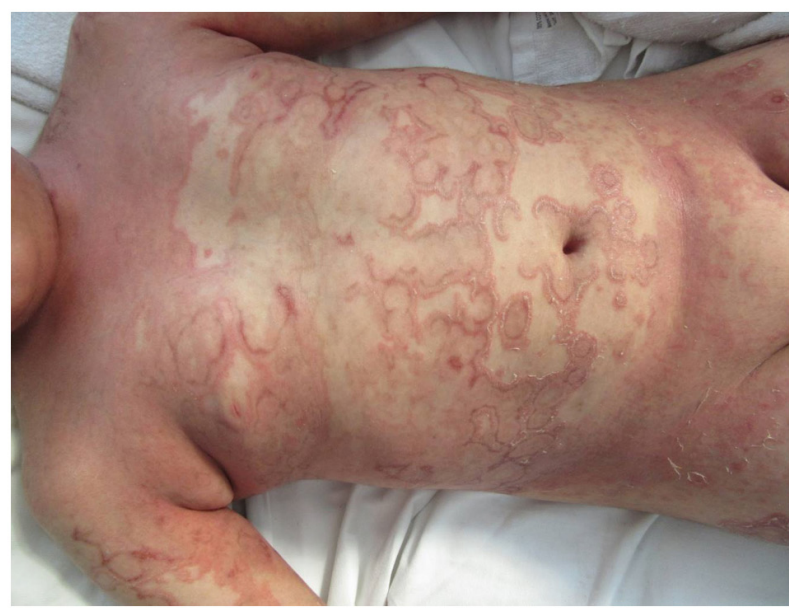

Figure 11. Pustular psoriasis.

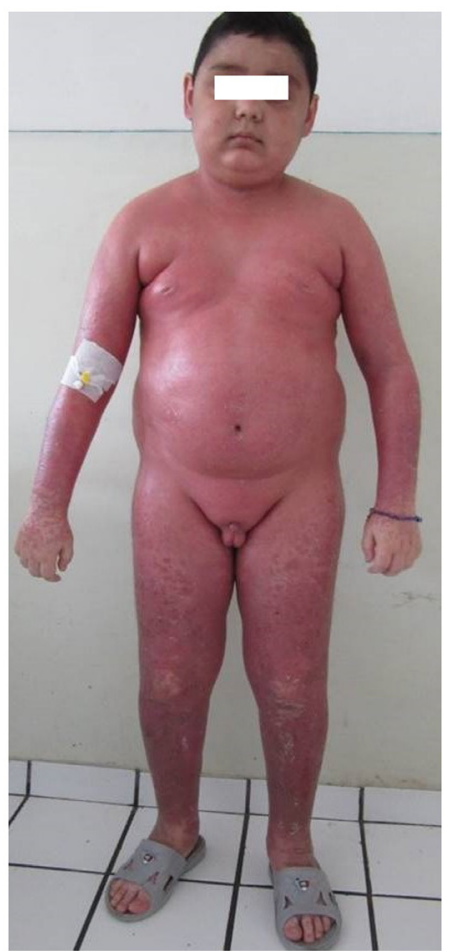

Figure 12. Erythrodermic psoriasis. Iatrogenic Cushing syndrome.

Vitamin D analogs, calcipotriene, and calcitriol inhibit the proliferation of keratinocytes and are safe to use.

Corticosteroids reduce inflammation and proliferation of keratinocytes, scaling, and erythema. Side effects of topical steroids are divided into local and systemic. Local side effects occur with prolonged treatment and are related to the corticosteroid potency and site of application (42). The most frequent side effects are atrophy, striae (Figs. 14 and 15) acne, rosacea, perioral dermatitis, and purpura. Patients can also experience less common side effects such as hypertrichosis, delayed wound healing, pigment alteration, and aggravation of skin infections (42). Systemic side effects are more common in long-term topical treatment with highly potent corticosteroids on large areas, thin skin, or inflamed surfaces. Children have a large body surface area as related to the body volume so they have 


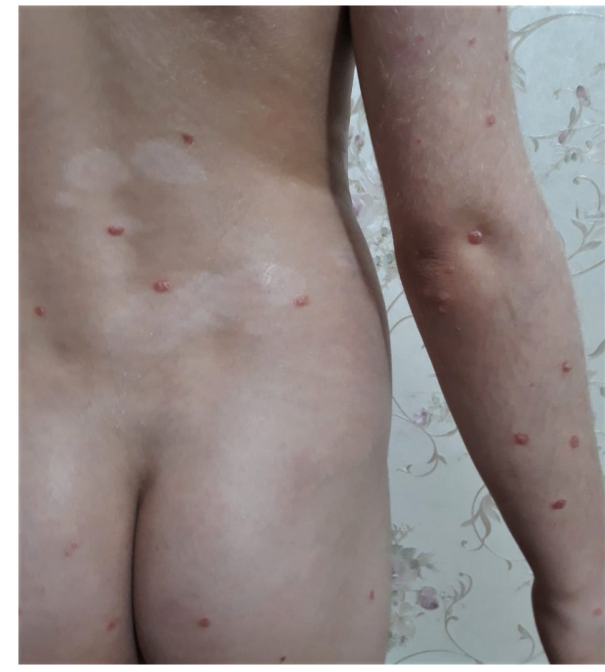

Figure 13. Psoriasis guttate and vitiligo.

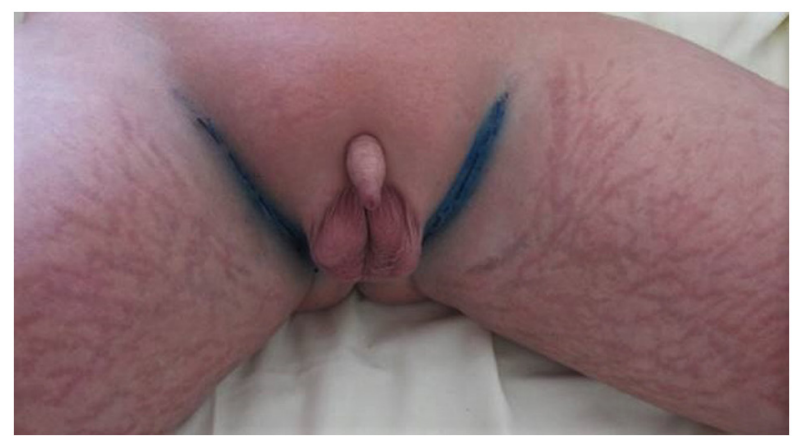

Figure 14. Post dermocorticoid striae in psoriasis.

a higher risk of developing hypothalamic-pituitary-adrenal axis suppression due to systemic absorption. Some systemic adverse effects are iatrogenic Cushing syndrome (moon face, centripetal obesity, striae) (Fig. 12), corticosteroid-related Addison crises, growth retardation (reduced bone mineral density, osteopathy), hyperglycemia and diabetes mellitus, edema, hypocalcemia, hypertension. During continued treatment, psoriatic patients may develop tachyphylaxis. Rebound phenomena may occur upon withdrawal of topic steroids applied on large areas of psoriasis for a long time. They are characterized by relapse or a papulopustular flare and may precipitate severe generalized pustular psoriasis (42).

Second-line topical treatments include retinoids, tars, anthralin, and keratolytics. Tazarotene decreases inflammation and helps to restore normal epidermal proliferation and differentiation (43). It is effective in the treatment of nail psoriasis. Tars have antipruritic and antiproliferative effect. Anthralin is especially used on thick plaques or large involved areas. Phenol and saline solution can be used for scalp lesions $(2,16)$.

Phototherapy. Phototherapy [narrow-band ultraviolet B (NB-UVB); psoralen plus ultraviolet A (PUVA)] is an alternative therapy for children with chronic plaque or guttate psoriasis that have an unsatisfactory result to topical treatment. Side effects of phototherapy in children include erythema, burning, hyperpigmentation, viral reactivation, and risk of cutaneous

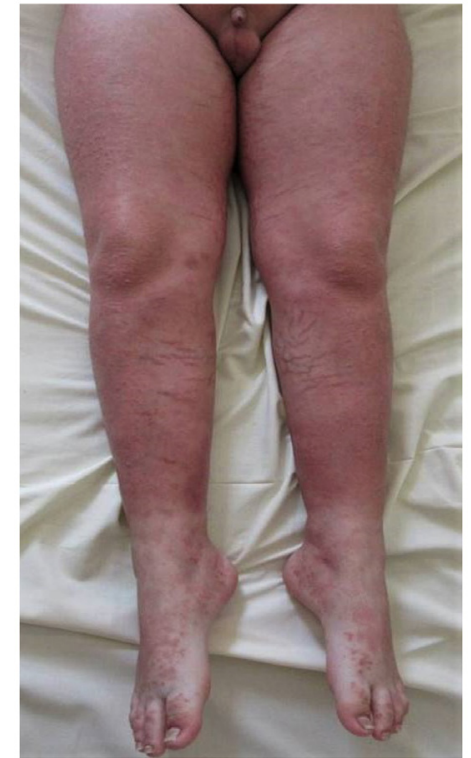

Figure 15. Post dermocorticoid striae in psoriasis.

carcinogenesis in long-term treatment with PUVA. Psoralen is avoided in children under 12 years of age. Phototherapy is contraindicated in children with cutaneous cancer syndromes or generalized erythroderma $(4,16)$.

Systemic therapy. Systemic therapy is required in moderate to severe disease. Methotrexate is the most common systemic medication used for psoriasis in children and it requires folic acid supplementation. The most serious side effects of methotrexate are bone marrow suppression, hepatic and pulmonary toxicity. Other options may include cyclosporine and systemic retinoids. Cyclosporin is well tolerated in pediatric patients and has a quick effect on severe, pustular, and erythrodermic psoriasis $(4,16,44-46)$.

Biologic therapy. Biologic therapy is becoming much more commonly used in pediatric patients. FDA has approved etanercept (TNF inhibitor) for patients 4 years and older, ustekinumab (IL-12 and IL-23 antagonist) for patients 12 years and older, and adalimumab (TNF inhibitor) was approved in Europe for patients 4 years and older (4). Etanercept is the favorite biologic therapy for pediatric patients and it was proved to be effective for moderate to severe psoriasis (15). It is a TNF inhibitor, which prevents activation of the inflammatory cascade. Studies have revealed that etanercept is more efficient and safe in long-term usage compared to other systemic treatments such as methotrexate, cyclosporine, and PUVA. Side effects include mild injection site reaction, increased risk of infections and cold-like illnesses, hepatitis B virus reactivation, and the possibility of weight gain $(47,48)$. Studies have shown that adalimumab is more efficient than methotrexate and has a similar safety profile (49). The advantage of adalimumab over other biologics consists in the efficacy in the treatment of psoriatic arthritis. Just like other TNF- $\alpha$ inhibitors, adalimumab has absolute contraindications to treatments such as tuberculosis and other severe infections (50). The precautions for the use of TNF- $\alpha$ inhibitors are hepatitis B infection, allergy, history of malignancy, and demyelinating conditions (51). Ustekinumab 
targets the IL-12 and IL-23 axis and it is efficient for psoriasis and psoriatic arthritis. A statistically significant increased response to ustekinumab in HLA-Cw6-positive patients has been noted. The different action mechanism of this drug provides an alternative treatment to TNF inhibitor failure, but there can be a certain problem for some patients regarding the slow onset of action (52). Some biological therapies are off-label (53). Infliximab has been approved for children with Crohn's disease, and several cases of induced psoriasis have been reported after a few weeks of therapy (54). Infliximab is used in limited cases such as for children with severe pustular psoriasis $(55,56)$. Certolizumab pegol is approved for psoriasis only in adults and it is currently being investigated in childhood arthritis and Crohn's disease (57,58). Secukinumab is not FDA-approved for any medical condition in children, but clinical trials are ongoing to evaluate the efficacy of this therapy in pediatric psoriasis $(59,60)$. Other biologics such as ixekizumab, guselkumab, and brodalumab, currently approved for adults with psoriasis, have been included in clinical trials for children with psoriasis (61-63).

\section{Conclusions}

Pediatric psoriasis is an increasing chronic skin disease, with exacerbations and remissions. The complexity of the pathology requires the involvement of a multidisciplinary team. Psychosocial support is an important part of psoriasis treatment due to the significant impact of the disease on the quality of life of the patients. Unfortunately, the lack of standardized treatment guidelines and the limited number of randomized clinical trials concerning biologic therapy make systemic treatment challenging in children with severe psoriasis.

\section{Acknowledgements}

Not applicable.

\section{Funding}

Publishing funds were supported by the Association of Dermatologists from Moldova.

\section{Availability of data and materials}

All information provided in this review is documented by relevant references.

\section{Authors' contributions}

DEB and DCB contributed to the study design, participated in the entire review process, and prepared the manuscript. SG, DB, ACN and CIB contributed to collecting the relevant literature, data analysis, and critical interpretation. RB, MAM, MG, ACP, AD and ILS conceived the concept of the review and modified the manuscript. All authors read and approved the final version of the manuscript for publication.

\section{Ethics approval and consent to participate}

Not applicable.

\section{Patient consent for publication}

A parental written informed consent before the publication of de-identified patient photographs was obtained.

\section{Competing interests}

All the authors declare that they have no competing interests.

\section{References}

1. Patterson JW: The psoriasiform reaction pattern. In: Weedon's Skin Pathology. 5th edition. Elsevier, Philadelphia, PA, pp99-120. e11, 2021.

2. Tollefson MM: Diagnosis and management of Psoriasis in children. Pediatr Clin North Am 61: 261-277, 2014

3. Kerkhof PCM van de and Nestlé FO: Psoriasis. In: Dermatology. 4th edtion. Philadelphia, PA, pp138-160, 2018.

4. Menter A, Cordoro KM, Davis DMR, Kroshinsky D, Paller AS, Armstrong AW, Connor C, Elewski BE, Gelfand JM, Gordon KB, et al: Joint American Academy of Dermatology-National Psoriasis Foundation guidelines of care for the management and treatment of psoriasis in pediatric patients. J Am Acad Dermatol 82: 161-201, 2020.

5. Tkach VY, Voloshynovych MS, Girnyk GY and Kozak NV: Clinical features and the course of psoriasis in children. Przegl Dermatol 107: 476-480, 2020.

6. Tollefson MM, Crowson CS, McEvoy MT and MaraditKremers H: Incidence of psoriasis in children: A population-based study. J Am Acad Dermatol 62: 979-987, 2010.

7. Murzina E: Pediatric Psoriasis: Clinical Features and Course. OAJBS 1: OAJBS.ID.000147, 2020.

8. Gelfand JM, Weinstein R, Porter SB, Neimann AL, Berlin JA and Margolis DJ: Prevalence and treatment of psoriasis in the United Kingdom: A population-based study. Arch Dermatol 141: 1537-1541, 2005.

9. Pinson R, Sotoodian B and Fiorillo L: Psoriasis in children. Psoriasis (Auckl) 6: 121-129, 2016.

10. Parisi R, Symmons DP, Griffiths CE and Ashcroft DM; Identification and Management of Psoriasis and Associated ComorbidiTy (IMPACT) project team: Global epidemiology of psoriasis: A systematic review of incidence and prevalence. J Invest Dermatol 133: 377-385, 2013.

11. Paller AS, Singh R, Cloutier M, Gauthier-Loiselle M, Emond B, Guérin A and Ganguli A: Prevalence of psoriasis in children and adolescents in the United States: A claims-based analysis. J Drugs Dermatol 17: 187-194, 2018.

12. Seyhan M, Coşkun BK, Sağlam H, Ozcan H and Karincaoğlu Y: Psoriasis in childhood and adolescence: Evaluation of demographic and clinical features. Pediatr Int 48: 525-530, 2006.

13. Kwon HH, Na SJ, Jo SJ and Youn JI: Epidemiology and clinical features of pediatric psoriasis in tertiary referral psoriasis clinic. J Dermatol 39: 260-264, 2012.

14. Ran D, Cai M and Zhang X: Genetics of psoriasis: A basis for precision medicine. Precis Clin Med 2: 120-130, 2019.

15. Paller AS, Siegfried EC, Pariser DM, Rice KC, Trivedi M, Iles J, Collier DH, Kricorian G and Langley RG: Long-term safety and efficacy of etanercept in children and adolescents with plaque psoriasis. J Am Acad Dermatol 74: 280-287.e1-e3, 2016.

16. Kliegman RM, Geme JWS, Blum NJ, Shah SS, Tasker RC and Wilson KM: Diseases of the epidermis. In: Nelson Textbook of Pediatrics. Kliegman RM and Geme JWS (eds). Elsevier, Philadelphia, PA, pp3501-3510.e1, 2020.

17. Rendon A and Schäkel K: Psoriasis pathogenesis and treatment. Int J Mol Sci 20: 1475, 2019.

18. Batani A, Brănișteanu DE, Ilie MA, Boda D, Ianosi S, Ianosi G and Caruntu C: Assessment of dermal papillary and microvascular parameters in psoriasis vulgaris using in vivo reflectance confocal microscopy. Exp Ther Med 15: 1241-1246, 2018.

19. Căruntu C, Boda D, Căruntu A, Rotaru M, Baderca F and Zurac S: In vivo imaging techniques for psoriatic lesions. Rom J Morphol Embryol 55 (Suppl 3): S1191-S1196, 2014.

20. Das RP, Jain AK and Ramesh V: Current concepts in the pathogenesis of psoriasis. Indian J Dermatol 54: 7-12, 2009.

21. Qing M, Liu P, Zhu W, Chen M, Chen M and Kuang Y: Analysis for 208 children with psoriasis vulgaris. Zhong Nan Da Xue Xue Bao Yi Xue Ban 45: 804-811, 2020 (In Chinese, English). 
22. Nguyen TT: Papulosquamous eruptions. In: Conn's Current Therapy 2021. Kellerman RD and Rakel D (eds). Elsevier, Philadelphia, PA, pp1056-1061, 2021.

23. Kim SK, Kang HY, Kim YC and Lee ES: Clinical comparison of psoriasis in Korean adults and children: Correlation with serum anti-streptolysin O titers. Arch Dermatol Res 302: 295-299, 2010.

24. Say M, Boralévi F, Lenormand C, Bursztejn AC, Estève E, Phan A, Bourrat E, Lacour JP, Richard MA, Acher A, et al: Clinical and therapeutic aspects of linear psoriasis: A study of 30 cases. Am J Clin Dermatol 19: 609-615, 2018.

25. Thomas L, Azad J and Takwale A: Management of nail psoriasis. Clin Exp Dermatol 46: 3-8, 2021.

26. Uber M, Carvalho VO, Abagge KT, Robl Imoto R and Werner B: Clinical features and nail clippings in 52 children with psoriasis Pediatr Dermatol 35: 202-207, 2018.

27. Narla S and Silverberg JI: 15838 Autoimmune comorbidities of psoriasis in US adults and children. J Am Acad Dermatol 83: AB48, 2020.

28. Brandon TG, Manos CK, Xiao R, Ogdie A and Weiss PF: Pediatric psoriatic arthritis: A population-based cohort study of risk factors for onset and subsequent risk of inflammatory comorbidities. J Psoriasis Psoriatic Arthritis 3: 131-136, 2018.

29. Thomas $\mathbf{J}$ and Parimalam K: Treating pediatric plaque psoriasis: Challenges and solutions. Pediatric Health Med Ther 7: 25-38, 2016

30. Caruntu C, Boda D, Dumitrascu G, Constantin C and Neagu M: Proteomics focusing on immune markers in psoriatic arthritis. Biomark Med 9: 513-528, 2015.

31. Brihan I, Hălmăjan A, Boda D, Ianosi SL, Fekete GL and Zdrîncă M: Role of osteodensitometry in osteoporosis and osteopenia in psoriatic arthritis. Exp Ther Med 20: 188, 2020.

32. Taranu T, Constantin M, Ungureanu D, Esanu IM and Toader MP IL6 is correlated with metabolic syndrome parameters in oral lichen planus. In: $2015 \mathrm{E}-\mathrm{Health}$ and Bioengineering Conference (EHB). pp1-4, 2015. http://toc.proceedings.com/29132webtoc.pdf

33. Brihan I, Ianoși SL, Boda D, Hălmăjan A, Zdrîncă M and Fekete LG: Implications of self-esteem in the quality of life in patients with psoriasis. Exp Ther Med 20: 202, 2020.

34. Gånemo A, Wahlgren CF and Svensson Å: Quality of life and clinical features in Swedish children with psoriasis. Pediatr Dermatol 28: 375-379, 2011.

35. Branisteanu DC, Stoleriu G, Branisteanu DE, Boda D, Branisteanu CI, Maranduca MA, Moraru A, Stanca HT, Zemba M and Balta F: Ocular cicatricial pemphigoid (Review). Exp Ther Med 20: 3379-3382, 2020.

36. Karabulut AA, Yalvac IS, Vahaboglu H, Nurozler AB and Duman S: Conjunctival impression cytology and tear-film changes in patients with psoriasis. Cornea 18: 544-548, 1999.

37. Kilic B, Dogan U, Parlak AH, Goksugur N, Polat M, Serin D and Ozmen S: Ocular findings in patients with psoriasis. Int J Dermatol 52: 554-559, 2013.

38. Cruz NFSD, Brandão LS, Cruz SFSD, Cruz SASD, Pires CAA and Carneiro FRO: Ocular manifestations of psoriasis. Arq Bras Oftalmol 81: 219-225, 2018.

39. Erbagci I, Erbagci Z, Gungor K and Bekir N: Ocular anterior segment pathologies and tear film changes in patients with psoriasis vulgaris. Acta Med Okayama 57: 299-303, 2003.

40. Salek SS, Pradeep A, Guly C, Ramanan AV and Rosenbaum JT: Uveitis and juvenile psoriatic arthritis or psoriasis. Am J Ophthalmol 185: 68-74, 2018

41. Mahé E: Childhood psoriasis. Eur J Dermatol 26: 537-548, 2016

42. Coondoo A, Phiske M, Verma S and Lahiri K: Side-effects of topical steroids: A long overdue revisit. Indian Dermatol Online J 5: 416-425, 2014

43. Ogawa E, Sato Y, Minagawa A and Okuyama R: Pathogenesis of psoriasis and development of treatment. J Dermatol 45: 264-272, 2018.

44. Negrei C, Ginghina O, Caruntu C, Burcea-Dragomiroiu GTA, Jinescu $\mathrm{G}$ and Boda D: Investigation relevance of methotrexate polyglutamates in biological systems by high performance liquid chromatography. REV CHIM (Bucharest) 66: 766-768, 2015.

45. Negrei C, Caruntu C, Ginghina O, Burcea-Dragomiroiu GTA, Toderescu CD and Boda D: Qualitative and quantitative determination of methotrexate polyglutamates in erythrocytes by high performance liquid chromatography. REV CHIM (Bucharest) 66 : 607-610, 2015
46. Boda D, Negrei C, Nicolescu F and Balalau C: Assessment of some oxidative stress parameters in methotrexate treated psoriasis patients. Farmacia 62: 704-710, 2014.

47. Nguyen TU and Koo J: Etanercept in the treatment of plaque psoriasis. Clin Cosmet Investig Dermatol 2: 77-84, 2009.

48. Branisteanu DE, Voicu CM, Cretu A, Dimitriu A, Luca MC and Salavastru CM: Adverse reactions of biological therapy for psoriasis. Rev Med Chir Soc Med Nat Iasi 119: 38-44, 2015

49. Papp K, Thaçi D, Marcoux D, Weibel L, Philipp S, Ghislain PD, Landells I, Hoeger P, Kotkin C, Unnebrink K, et al: Efficacy and safety of adalimumab every other week versus methotrexate once weekly in children and adolescents with severe chronic plaque psoriasis: A randomised, double-blind, phase 3 trial. Lancet 390: 40-49, 2017.

50. Khobzey K, Liskova I, Szegedi A, Pavlovsky L, Lunder T, Kingo K, Miljković J, Péč J, Bohinc M and Hojnik M: Effectiveness of adalimumab in the treatment of scalp and nail affection in patients with moderate to severe plaque psoriasis in routine clinical practice. Acta Dermatovenerol Alp Pannonica Adriat 26: 11-14, 2017.

51. Mocanu M, Toader MP, Rezus E and Taranu T: Aspects concerning patient adherence to anti-TNF $\alpha$ therapy in psoriasis: A decade of clinical experience. Exp Ther Med 18: 4987-4992, 2019.

52. Savage LJ, Wittmann M, McGonagle D and Helliwell PS: Ustekinumab in the treatment of psoriasis and psoriatic arthritis. Rheumatol Ther 2: 1-16, 2015.

53. Cline A, Bartos GJ, Strowd LC and Feldman SR: Biologic treatment options for pediatric psoriasis and atopic dermatitis. Children (Basel) 6: 103, 2019.

54. Broge T, Nguyen N, Sacks A and Davis M: Infliximab-associated psoriasis in children with Crohn's disease may require withdrawal of anti-tumor necrosis factor therapy. Inflamm Bowel Dis 19: E75-E77, 2013.

55. Menter MA and Cush JM: Successful treatment of pediatric psoriasis with infliximab. Pediatr Dermatol 21: 87-88, 2004.

56. Skrabl-Baumgartner A, Weger W, Salmhofer W and Jahnel J: Childhood generalized pustular psoriasis: Longtime remission with combined infliximab and methotrexate treatment. Pediatr Dermatol 32: e13-e14, 2015.

57. UCB Celltech: (2018). The Use of Certolizumab Pegol for Treatment of Active Crohn's Disease in Children and Adolescents (NURTURE). ClinicalTrials.gov Identifier: NCT00899678. https://clinicaltrials.gov/ct2/show/NCT00899678.

58. UCB BIOSCIENCES GmbH: (2020). Pediatric Arthritis Study of Certolizumab Pegol (PASCAL). ClinicalTrials.gov Identifier: NCT01550003.https://clinicaltrials.gov/ct2/show/NCT01550003.

59. Novartis Pharmaceuticals: (2020). Pediatric Study in Children and Adolescents With Severe Plaque Psoriasis. ClinicalTrials. gov Identifier: NCT02471144. https://clinicaltrials.gov/ct2/show/ NCT02471144

60. Novartis Pharmaceuticals: (2020). Study to Assess the Long-term Safety, Tolerability, Efficacy of Secukinumab in Pediatric Patients of Age 6 to $<18$ Years, With Moderate to Severe Plaque Psoriasis. ClinicalTrials.gov Identifier: NCT03668613. https://clinicaltrials. gov/ct2/show/NCT03668613.

61. Eli Lilly and Company: (2020). Study of Ixekizumab (LY2439821) in Children 6 to Less Than 18 Years With Moderate-to-Severe Plaque Psoriasis (Ixora-peds). ClinicalTrials.gov Identifier: NCT03073200. https://clinicaltrials.gov/ct2/show/NCT03073200

62. Janssen Research \& Development, LLC: (2020). A Study to Evaluate the Efficacy, Safety, and Pharmacokinetics of Subcutaneously Administered Guselkumab for the Treatment of Chronic Plaque Psoriasis in Pediatric Participants (PROTOSTAR). ClinicalTrials.gov Identifier: NCT03451851. https://clinicaltrials. gov/ct2/show/NCT03451851.

63. Bausch Health Americas, Inc. (2021). An Open-label, Single-dose Study to Evaluate Safety, Tolerability, and Pharmacokinetics of Brodalumab in Pediatric Subjects. ClinicalTrials.gov Identifier: NCT03240809. https://clinicaltrials.gov/ct2/show/NCT03240809.

This work is licensed under a Creative Commons Attribution-NonCommercial-NoDerivatives 4.0 International (CC BY-NC-ND 4.0) License. 